\title{
Invaginated Graft Insertion Anastomosis for a Type Ib Endoleak at the Supraceliac Aorta Following Thoracic Endovascular Aortic Repair
}

\author{
Takashi Yamauchi, MD, PhD, Suguru Kubota, MD, PhD, and Kosei Hasegawa, MD
}

\begin{abstract}
Open surgery for a type Ib endoleak after thoracic endovascular aortic repair is associated with some knacks and pitfalls, particularly in cases wherein the distal edge of a stent graft is located at just supraceliac aorta and the renovisceral segment and infrarenal aorta do not require surgical intervention. Here we describe the invaginated graft insertion technique to provide an easy and secure anastomosis in such clinical situations. This procedure may be feasible for anastomosis between a prosthetic vascular graft and a previously deployed stent graft when the location of the anastomosis is distant from the surface of the skin incision with a narrow and limited surgical view.
\end{abstract}

Keywords: thoracic endovascular surgery, endoleak, invaginated graft

\section{Introduction}

With the widespread use of thoracic endovascular aortic repair (TEVAR) in treatments, the number of cases requiring reintervention is increasing. In cases of a type $\mathrm{Ib}$ endoleak in which the distal edge of a stent graft is just above the main abdominal visceral artery, open surgery is recommended because of the lack of a sufficient landing zone for an additional stent graft. In such clinical situations, the surgical view is occasionally limited and distant from the surface of the skin incision with a size discrepancy between a previously deployed stent graft and the native aortic wall. A secure aortic clamp and an anastomosis between a new prosthetic vascular graft and an enlarged native aortic wall surrounding a previous endovascular stent graft have associated knacks and pitfalls.

Department of Cardiovascular Surgery, KKR Sapporo Medical Center, Sapporo, Hokkaido, Japan

Received: November 21, 2016; Accepted: January 17, 2017 Corresponding author: Takashi Yamauchi, MD, PhD. Department of Cardiovascular Surgery, KKR Sapporo Medical Center, 6-3-40 Ichijo Hiragishi, Toyohira, Sapporo, Hokkaido 062-0931, Japan

Tel: +81-11-822-1811, Fax: +81-11-841-4572

E-mail: yamataka@surg1.med.osaka-u.ac.jp

\section{Case Report}

A 61-year-old male presented with a past history of TEVAR (GORE TAG thoracic endoprosthesis; $37 \mathrm{~mm} \times$ $20 \mathrm{~cm}, 40 \mathrm{~mm} \times 20 \mathrm{~cm}$; W. L. Gore and Associates) for a true aneurysm of the descending aorta 4 years prior. In addition, he had a past history of tuberculosis and right nephrectomy for renal cell carcinoma. The diameter of the native aorta at the distal end of the stent graft was $38 \mathrm{~mm}$ at the time. Subsequently, the diameter of the aorta at the end of the thoracic stent graft gradually increased, and the diameter of the native aortic wall exceeded the size of the stent graft 2 years later. A type Ib endoleak and descending aneurysm enlargement were not observed because of thrombosis between the aorta and the stent graft. The patient refused surgical intervention, and a careful follow-up was continued. However, a type Ib endoleak occurred 4 years later because of enlargement of the aorta at the diaphragm (from $38 \mathrm{~mm}$ to $58 \mathrm{~mm}$; Fig. 1A) associated with an enlargement of the middle descending aorta and onset of consumptive disseminated intravascular coagulation (DIC). His DIC score was 6 , as defined by the International Society on Thrombosis and Hemostasis. The distance between the distal edge of the stent graft and the celiac artery was less than $17 \mathrm{~mm}$ (Fig. 1B). Moreover, the ostium of the celiac artery and superior mesenteric artery was very close, and the aortic diameter below the celiac artery was approximately $30 \mathrm{~mm}$. We considered TEVAR with a fenestrated stent graft or celiac artery coverage as treatment options; however, long-term results remained unclear with technical difficulties. Therefore, we planned graft replacement between just above the celiac artery and the distal edge of the stent graft.

Graft replacement of the entire descending aorta was not chosen because severe adhesion in the upper left pleural cavity was predicted because of the past history of tuberculosis. The patient was placed in the right lateral decubitus position, and a left thoracoabdominal incision was made through the seventh intercostal space. The abdominal section was approached via the retroperitoneal space, and the aorta was exposed at the level of the supe- 
A)

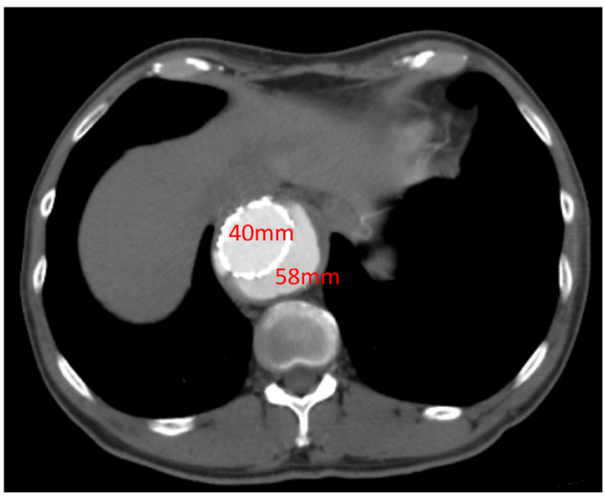

B)

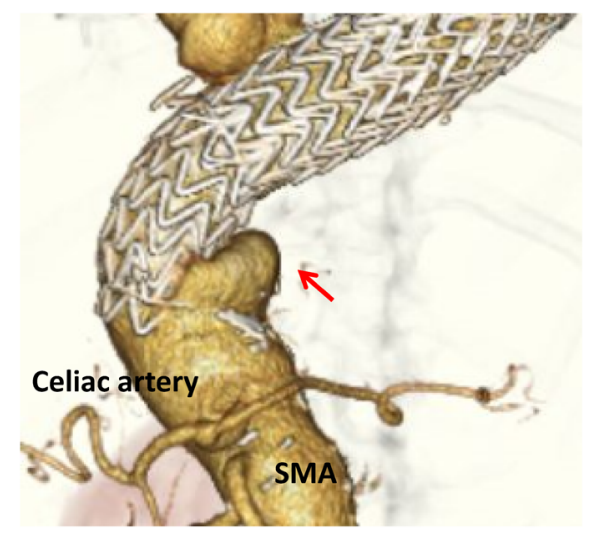

Fig. 1 (A) Diameters of the native aorta and the distal edge of the stent graft were $40 \mathrm{~mm}$ and $58 \mathrm{~mm}$, respectively. (B) A type lb endoleak (arrow) occurred because of an enlargement of the native aorta after TEVAR. The distance between the distal edge of the stent graft and the celiac artery was $17 \mathrm{~mm}$. In addition, the ostium of the celiac artery and superior mesenteric artery was very close. TEVAR: thoracic endovascular aortic repair; SMA: superior mesenteric artery

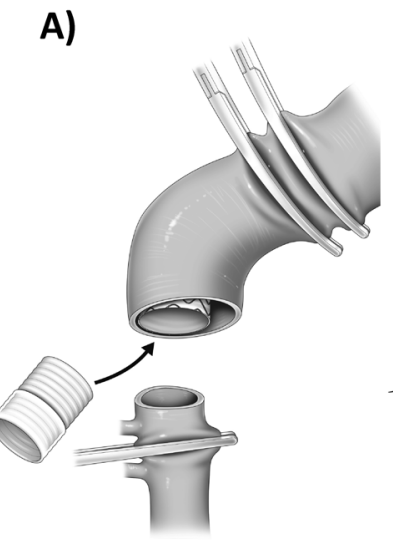

B)

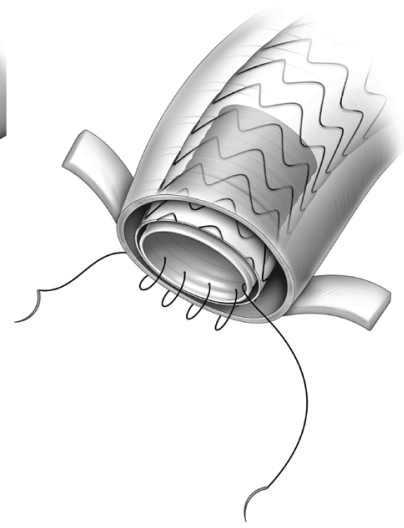

C)

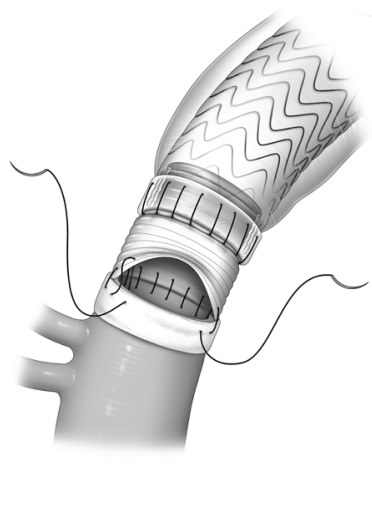

Fig. 2 (A) The proximal aorta was clamped using two large arcuate forceps at the distal descending aorta. An additional clamp was placed between the celiac artery and the superior mesenteric artery. (B) An invaginated tube graft was inserted into the previously deployed stent graft. The anastomosis was performed using an over-and-over suture with 3-0 PROLENE, reinforced by a Teflon ${ }^{\mathrm{TM}}$ felt strip, and adjusted for any size discrepancy. (C) Distal anastomosis between the native aortic wall and the new prosthetic graft was performed using an over-and-over suture with 4-0 PROLENE.

rior mesenteric artery. A femoral-femoral extracorporeal bypass was established. Heparin $(300 \mathrm{U} / \mathrm{kg})$ was administered, and the activated coagulation time was maintained above $400 \mathrm{~s}$. The pump flow was $1.5 \mathrm{~L} / \mathrm{min}$, and the systolic upper body blood pressure was maintained above $100 \mathrm{mmHg}$. The proximal aorta was $7 \mathrm{~cm}$ in diameter and was clamped using two large arcuate forceps at the distal descending aorta. Distal clamping was performed between the celiac artery and the superior mesenteric artery (Fig. 2A). The proximal anastomosis was as follows: 1) the flared stent of the distal end of the stent graft that appeared to make the proximal anastomosis difficult was cut first and 2) an invaginated $28 \mathrm{~mm}$ J graft (Japan Lifeline, Tokyo, Japan) was inserted into the previously deployed stent graft (Fig. 2B). There was a size discrepancy between the stent graft $(40 \mathrm{~mm}$ in diameter) and the native thickening of the aortic wall $(58 \mathrm{~mm}$ in diameter). Anastomosis was performed using an over-and-over suture with 3-0 PROLENE ${ }^{\circledR}$ (Ethicon. Inc., Somerville, NJ, USA), reinforced by a Teflon ${ }^{\mathrm{TM}}$ felt strip, adjusting the size discrepancy. This procedure could be easily performed even with the limited and distant surgical view. Some ad- 
A)

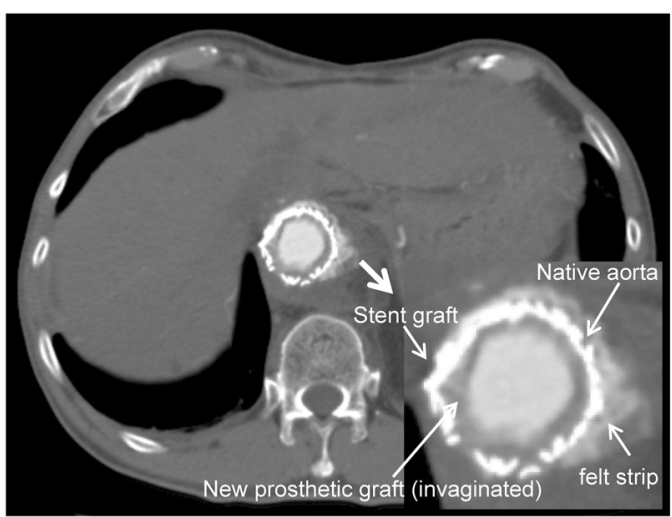

B)

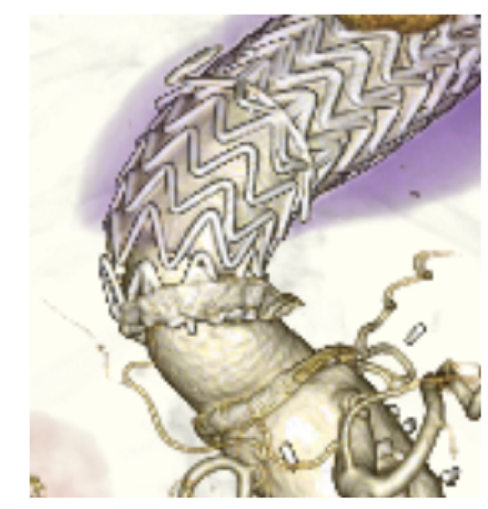

Fig. 3 (A) Postoperative CT findings at the proximal anastomosis site. (B) A short prosthetic graft was anastomosed, and no endoleak was observed. CT: computed tomography

ditional suturing was performed to secure hemostasis. The distal end of the inserted graft was proximally extracted, and the cut was adjusted for distal anastomosis. Distal anastomosis between the native aortic wall and the new prosthetic graft was performed using an over-and-over suture with 4-0 PROLENE ${ }^{\circledR}$ (Ethicon. Inc.) (Fig. 2C). There was no bleeding at the proximal anastomotic site. The postoperative computed tomography (CT) image is presented in Fig. 3.

\section{Discussion}

Among the reinterventions following TEVAR that covered the descending aorta at the level of the supraceliac artery, a type Ib endoleak is not a major concern; however, the condition does exist at a consistent rate. ${ }^{1,2)}$ In such cases, celiac coverage TEVAR might be a treatment option; however, it is often associated with an unsatisfactory outcome. ${ }^{2)}$ TEVAR with a fenestrated stent graft is accompanied by technical difficulties, and long-term results are limited. ${ }^{3)}$ Among these cases, open surgery is required because of the short distance between the main abdominal visceral branch artery and the distal end of the stent graft.

Replacement of the entire descending aorta is a treatment option that is occasionally difficult due to the patient's condition, such as chronic obstructive pulmonary disease, inflammatory disease, or past history of left thoracotomy. Therefore, graft replacement between the distal edge of the stent graft and the aorta just above the celiac artery is also an option; however, this procedure appears to be associated with some pitfalls. The first pitfall is the difficulty in performing a secure anastomosis. The location of the anastomosis is relatively distant from the surface of the skin incision and the range of the graft replacement is short, providing a limited surgical view. In addition, there is a significant size discrepancy between the native aortic wall and the stent graft. The presented invaginated graft insertion technique simply contributes to the proximal anastomosis, and the $1-2 \mathrm{~cm}$ anastomotic portion of the invaginated vascular graft plays an important role in reinforcement anastomosis with an occasionally fragile native aortic wall and a thin stent graft wall. This maneuver is similar to stepwise distal anastomosis for an arch aneurysm. ${ }^{4)}$ The presented technique also appears to be similar to that used in the open repair of the descending thoracic aorta following the elephant trunk technique. In the present method, the proximal anastomosis must be conducted between a stent graft and the native aortic wall with a size discrepancy in a limited surgical view. In contrast, after the elephant trunk technique, the prosthetic graft is associated with a wide surgical field. If the size discrepancy of the aortic wall and the underlying endovascular stent is substantial, the aortic wall should be tapered, as reported previously.5)

Another pitfall is proximal aortic clamping. Direct aortic clamping might result in insufficient aortic clamping because an aortic aneurysm underlying a stent graft is large and the thickening of the aortic wall with a massive thrombus in the false lumen is often large and hard. In the present study, two forceps were required for complete clamping. Moreover, an aortic occlusion balloon must be prepared for incomplete direct clamping using forceps. Therefore, a proximal clamping should be performed sufficiently distal from the proximal anastomotic site to ensure that there is sufficient space for the insertion of an occlusion balloon. In addition, direct clamping is associated with the possibility of injuring the stent graft structure or graft wall. Such an injury could result in disastrous complications such as an endoleak from the stent graft and rupture or dissection of the native aortic wall. Prior to direct clamping, we consulted the manufacturer of the stent graft regarding its safety. Johnston et al. reported no 
apparent injury to the TEVAR graft (Gore TAG and Cook Zenith TX2) by occluding the aorta underlying the endovascular stent with Fogarty-Hydragrip (Edwards Lifesciences, Irvine, CA, USA) in staged hybrid repair using a proximal endovascular aneurysm for the treatment of an extensive thoracoabdominal aortic aneurysm. ${ }^{5)}$

There might be an increase in the number of open surgical conversion cases for a type Ib endoleak following TEVAR. The presented method suggests a feasible surgical option in such clinical situations.

\section{Disclosure Statement}

The authors have no conflict of interest.

\section{Author Contributions}

Writing: TY

Critical review and revision: all authors

Final approval of the article: all authors
Accountability for all aspects of the work: all authors

\section{References}

1) Ehrlich MP, Rousseau H, Heijmen R, et al. Midterm results after endovascular treatment of acute, complicated type $\mathrm{b}$ aortic dissection: the talent thoracic registry. J Thorac Cardiovasc Surg 2013; 145: 159-65.

2) Rose MK, Pearce BJ, Matthews TC, et al. Outcomes after celiac artery coverage during thoracic endovascular aortic aneurysm repair. J Vasc Surg 2015; 62: 36-42.

3) Mastracci TM, Eagleton MJ, Kuramochi Y, et al. Twelveyear results of fenestrated endografts for juxtarenal and group IV thoracoabdominal aneurysms. J Vasc Surg 2015; 61: 355-64.

4) Ogino $\mathrm{H}$, Ando $\mathrm{M}$, Sasaki $\mathrm{H}$, et al. Total arch replacement using a stepwise distal anastomosis for arch aneurysms with distal extension. Eur J Cardiothorac Surg 2006; 29: 255-7.

5) Johnston WF, Upchurch GR Jr, Tracci MC, et al. Staged hybrid approach using proximal thoracic endovascular aneurysm repair and distal open repair for the treatment of extensive thoracoabdominal aortic aneurysms. J Vasc Surg 2012; 56: 1495-502. 